REM sleep control in the intact animal. When the connections between the pons and forebrain are severed, the pons and medulla alone can generate a REM sleep state, whereas no REM sleep is seen in the forebrain. Conversely, when the pons is connected to the forebrain and all connections between it and the medulla are severed, a REM sleep state is observed in the forebrain, but no such state is found in the medulla ${ }^{3}$. Lu et al. ${ }^{1}$ identify the extended part of the ventrolateral preoptic nucleus in the hypothalamus ${ }^{10}$ as a region that can inactivate the lateral pontine and ventral periaqueductal gray REM-off regions, thereby releasing the SLD REM-on region from inhibition and triggering REM sleep. In opposition to this inhibition of REM-off cells, they propose that the hypocretin neurons of the hypothalamus, which are active during certain waking behaviors but inactive in REM sleep ${ }^{11}$, project to the same REM-off neurons, but because hypocretin is excitatory, block REM sleep.

An understanding of REM sleep control is central to an understanding of sleep disorders, particularly the pathological signs seen in narcolepsy. Narcolepsy is characterized by excessive daytime sleepiness and sudden losses of muscle tone in alert waking (cataplexy), caused, in at least most cases, by a loss of hypocretin cells ${ }^{12}$. Lu et al. ${ }^{1}$ explain aspects of this syndrome, particularly the relatively rapid onset of REM sleep in these patients during sleep periods, in terms of the instability of the flip-flop switch. However, this does not completely explain cataplexy. In the normal individual, all noradrenergic locus coeruleus and histaminergic cells are active in waking and inactive in sleep, particularly in REM sleep. In cataplexy, the loss of the excitatory hypocretin input that maintains noradrenergic activity during certain emotions allows noradrenergic activity to cease in waking, while histaminergic activity persists ${ }^{13}$. The disfacilitation of motoneurons resulting from the loss of noradrenergic input contributes to the loss of muscle tone, whereas the maintained discharge of histamine cells allows maintenance of consciousness, the defining criteria of cataplexy.

Neuronal recording studies in the cat and dog have identified a population of neurons in the medial medulla of the cat and dog that are active only during periods of muscle tone suppression $^{14}$, that is, REM sleep and cataplexy. Stimulation of these cells suppresses muscle tone by release of GABA and glycine, and lesioning this region reduces the normal muscle tone suppression of REM sleep ${ }^{3}$. Activity in this inhibitory cell group is synaptically linked to inactivity in locus coeruleus cells, which when active help maintain muscle tone $\mathrm{e}^{3,13,14}$. Lu et al. find that large medullary lesions do not prevent muscle tone suppression in the rat, so they hypothesize that direct connections from the pons to glycinergic interneurons in the ventral horn are responsible for muscle tone suppression in the rat. This could represent a species difference between dogs, cats and rats. However, previous work ${ }^{5}$ identified projections in the rat from the SLD to the same medial medullary regions identified in the cat and dog. The lesion placement by Lu et al. (see Fig. $4 \mathrm{~d}$ in their paper) is rostral to the region that other studies have identified as the medullary relay for muscle tone suppression ${ }^{15}$. Therefore, it remains possible that the medullary inhibitory region is similar in the rat, cat and dog.

Finally, there is the question of "why do we dream," or the physiological correlate of the question, "why do we have REM sleep?" Although physiologists have made considerable progress in localizing the cells critical for REM sleep control, we have not yet been able to use this knowledge to answer the fundamental question of why this state exists. The search for the control of REM sleep can become an infinite regression; if REM-on cells trigger REM sleep, then what triggers or disinhibits the REM-on cells? What then controls this latter cell population? One would like to see evidence that REM sleep is initiated to accomplish some task and terminated when that task is completed. Many ideas for the nature of this task have been offered, from brain warming, to the upregulation of certain classes of receptors, to arousal to allow surveillance of the environment ${ }^{2}$. The discovery of the ultimate driving mechanism(s) of REM sleep will undoubtedly be facilitated by studies in the rat using these pioneering techniques ${ }^{1,5}$.

1. Lu, J., Sherman, D., Devor, M. \& Saper, C.B. Nature (2006) published online 10 May 2006 (doi:10.1038/ nature04767)

2. Siegel, J.M. Nature 437, 1264-1271 (2005)

3. Siegel, J.M. in Principles and Practice of Sleep Medicine (eds. Kryger, M.H., Roth, T. \& Dement, W.C.) 120-135 (Elsevier Saunders, Philadelphia, 2005).

4. Sakai, K., Crochet, S. \& Onoe, H. Arch. Ital. Biol. 139 93-107 (2001).

5. Boissard, R. et al. Eur. J. Neurosci. 16, 1959-1973 (2002).

6. Karczmar, A.G., Longo, V.G. \& Scotti, D.C. Physiol. Behav. 5, 175-182 (1970)

7. Kodama, T., Lai, Y.Y. \& Siegel, J.M. Brain Res. $\mathbf{5 8 0}$ 348-350 (1992)

8. Benington, J.H., Kodali, S.K. \& Heller, H.C. Sleep 17 28-36 (1994).

9. McGinty, D.J. \& Siegel, J.M. Prog. Psychobiol. Physiol. Psychol. 15, 85-140 (1992).

10. Gvilia, I., Turner, A., McGinty, D. \& Szymusiak, R. J. Neurosci. 26, 3037-3044 (2006).

11. Mileykovskiy, B.Y., Kiyashchenko, L.I. \& Siegel, J.M Neuron 46, 787-798 (2005)

12. Thannickal, T.C. et al. Neuron 27, 469-474 (2000).

13. John, J., Wu, M.-F., Boehmer, L.N. \& Siegel, J.M. Neuron 42, 619-634 (2004).

14. Siegel, J.M. et al. Science 252, 1315-1318 (1991).

15. Lai, Y.Y. et al. J. Comp. Neurol. 408, 419-436 (1999)

\title{
More neurons may not make you smarter
}

The hippocampus continues to add new neurons even in adulthood. When animals are housed in 'enriched' or stimulating environments with opportunities for exercise and/or games, they show greater neurogenesis in the hippocampus. Animals exposed to these more complex cages also show improvements in several tasks of memory and anxiety.

A study by René Hen and colleagues on page 729 reports, however, that there is no link between the increased neurogenesis that comes with enriched environments and improvements in the memory tasks. The authors used a focused dose of radiation to prevent neurogenesis selectively in the hippocampus of mice before placing them in enriched cages. When tested six weeks later on an anxiety and spatial memory task, these animals still did better than animals housed in standard cages, suggesting that the lack of neurogenesis did not matter to their learning ability.

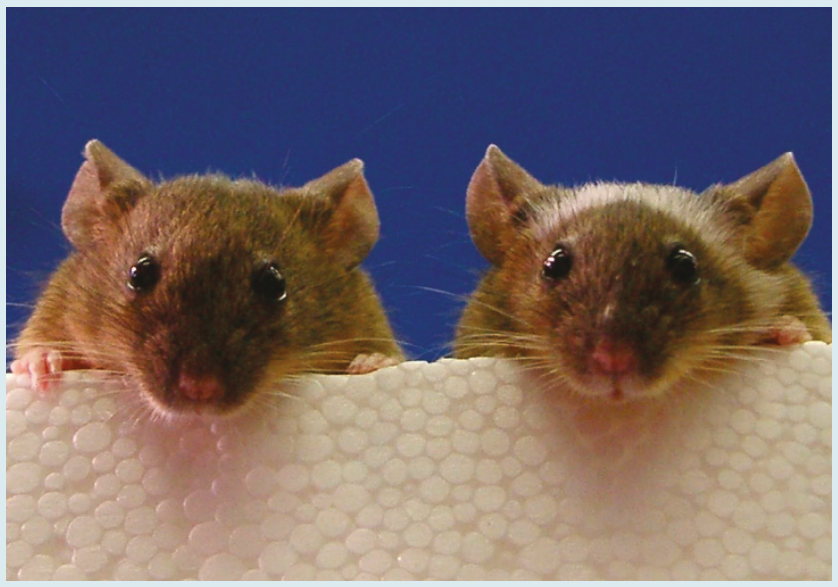

